\title{
Exhaustion of Resources: a Marked Temporal Process
}

\section{Framework}

\author{
Roy Cerqueti \\ University of Macerata - Department of Economics and Law \\ Via Crescimbeni, 20 - 62100 - Macerata, Italy. \\ Tel.: +390733258 3246 - Fax: +3907332583205 \\ E-mail: roy.cerqueti@unimc.it
}

July 30, 2013

\begin{abstract}
This paper deals with the exhaustion of a replenishable resource, one of the main topics in environmental research. To examine this problem, we construct a mechanism to estimate the probability of depletion under a very small set of assumptions. The stock of the resource is assumed to evolve accordingly to a marked temporal process generated by the shocks occurring in the environment. Our setting is eminently theoretical, and constitutes an effective extension of some important models of stochastic growth regarding sustainability and ruin analysis. Furthermore, the validity of the model is supported through the evidence relative to the paradigmatic cases of water resource planning and oil reserves.
\end{abstract}

Keywords: Stochastic growth; resource exhaustion; Spatial Mixed Poisson Process; Sustainability.

\section{Introduction}

Sustainability represents nowadays one of the most challenging targets to be achieved by policymakers. The goal of sustainable policies is to avoid the irretrievable depletion of resources.

This paper explores this problem by analyzing a case of exhaustion of a replenishable ${ }^{1}$ resource, named R.

To study the case, we need to establish how the available stock of $R$ evolves over time. At this aim, the identification of the scientific context of the present research is needed. This will be done by discussing the paradigmatic cases of drinking-water and fossil fuels, which are replenishable resources

\footnotetext{
${ }^{1}$ The term replenishable must be here intended in the sense of availability. In this respect, a resource is said to be replenished when an additional stock of it becomes available.
} 
whose availability has been the focus of a wide number of environmental researches. We will then elaborate on these examples, in order to clarify the scientific basis of the present paper.

The shortage of water in arid regions normally requires the implementation of water resource planning. In order to address this, a policymaker needs to find a balance between consumption due to human activities and preserving water reserves. This task is quite complicated also due to the presence of uncertainty in the water resource system.

More specifically, stochastic uncertainties make it impossible to base water resource management models on conventional deterministic quantitative methods ( $\mathrm{Li}$ et al. 2006, Chen et al. 2013): Anderson et al. (2000) address this problem by including also stochastic factors which affect the available stock of the resource; Guo and Huang (2009), Lv et al. (2012), Maqsood et al. (2005), Hu et al. (2012), Gu et al. (2013) treat the uncertainty associated to the water resource by adopting techniques from fuzzy sets theory; Sullivan (2011) discusses the uncertainty and introduces the concept of water vulnerability.

A related strand of literature has concentrated on identifying the factors whose impact affects water availability. In this respect, it is commonly acknowledged that climate changes and human activities play a relevant role. The impact of human activities and climate changes on water resources has been explored from several perspectives (e.g.: Loukas et al. 2002; Jones and Post 2004; Tamerius et al. 2006; Wang et al. 2006; Zhang et al. 2010; Yu et al. 2011). However, a definitive quantification of such impact seems yet to be achieved. It is worth citing some recent contributions, which analyze the reaction of a water resource system and, more in general, of an ecosystem to exogenous events of natural or human origin (e.g.: Ouyang et al. 2000, Herrera-Silveira and Morales-Ojeda 2009, Dearborn and Kark 2010, Li et al. 2010, Dai et al. 2012).

In general, policymakers are interested in knowing the expected availability of water, in order to choose the best strategies in water resource planning (Hoppe et al. 2004).

For what concerns fossil fuels, we present some empirical evidence. Table 1 collects data from the OPEC Annual Statistical Bulletin 2010/2011.

\section{INSERT TABLE 1 ABOUT HERE}

Data exhibit the presence of jumps in the dynamics of the stock of available oil reserves, which is worth examining in more detail. Figure 1 is a plot of the oil reserves (billions of barrels) in Venezuela and Iran in the 1980-2010 period.

\section{INSERT FIGURE 1 ABOUT HERE}

Venezuela experienced jumps in oil reserves in 1985, 2008, 2009 and 2010. It is important to identify the causes of such jumps. First, we may note that in the mid-1980's the price of oil began to fall, due to the increase in production decided by OPEC members. The jumps in 2008-2010 period are probably associated with huge investments by the Venezuelan Government in the exploration of the 
oilfields of the Orinoco Basin.

Oil reserves in Iran, as in Venezuela, experienced a jump in the mid-1980's due to the abovementioned decision by the OPEC members. The jump in 2002 might be due to the discovery in the region of Khuzestan of the oilfield Yadavaran, which is actually composed of the oilfield Koushk (discovered in 2000) and the oilfield Hosseinieh (discovered in 2002), and contains at least 17 billions of barrels of oil.

Some facts emerge from studies on water and oil: first, the nature of the evolution of the stock of a resource seems to be random and of jump-type; second, a jump in the stock is a consequence of an event occurring in the (natural and/or human) context where the resource is located; third, the evolution of the stock of the resource must be fully understood to perform resource planning policies. In this paper we include all these arguments within a theoretical model, which refers to a generic replenishable resource. The model can be easily applied to water resource planning or oil reserves. Specifically, we construct a stochastic growth model describing the dynamics of $\mathrm{R}$ and then provide an estimate of the stock of resource available at a fixed future date. At this aim, we assume the knowledge of some elements of the previous history of the stock of resource.

We stress once again that, based on the evidence, the future stock of resources depends on the exogenous shocks occurring in the environment. A-priori, the shocks appear at random times, and their impact on the available stock of $\mathrm{R}$ is random as well.

In details, our approach can be then summarized as following:

(i) In the first step, we define a stochastic process which represents the quantitative translation of the (times and marks of the) qualitative exogenous shocks occurring in the environment.

(ii) The second step consists in deriving the dynamics of the available resource stock through the definition of a suitable transformation of the process of the shocks in step $(i)$.

(iii) In the third step, an estimation of the stock of $\mathrm{R}$ accumulated at a (future) date is provided.

(iv) As fourth step, some insights regarding the depletion of the resource within a time interval are provided.

In step $(i)$, the stochastic process related to the exogenous shocks is introduced. In particular, we adopt a Spatial Mixed Poisson Process (SMPP) framework, which constitutes a generalization of the standard Poisson Spatial Processes (see the Appendix for the Definition and some useful results). By dealing with SMPPs we obtain a very general -even if rather complex- model. Indeed, as we will see in the discussion following Assumption 2, through SMPPs we are able to describe realistically the dynamics of the available resource stock. Moreover, the stochastic structure of SMPPs allows to capture also the occurrence of rare events which may affect the dynamics of the stock. In this respect, we provide a theoretical answer to the question posed by Ludwig (1999) on the validity 
of the depletion probability estimations. As a further positive aspect of the SMPP framework, we mention that this general set up trivially contains all the models of Markovian type as remarkable subcases. The most prominent example is Mitra and Roy (2007), which describes the evolution of a replenishable resource in a discrete deterministic time setting through a distorted Markovian process, by adopting a sequence of i.i.d. random variables and a particular one-step transition function. As for the description of the dynamics of the stock of the resource, our reference paper is Mitra and Roy (2007). Indeed, it is the most recent relevant contribution to the field of stochastic growth applied to the evolution of the resources. In particular, it is worth noting that Mitra and Roy's model represents an extension of the important contribution of Athreya (2003), mainly for what concerns the assumptions on the transition function. A different perspective is provided by Nishimura et al. (2006), who develop a Markovian model in presence of exogenous shocks -with possible unbounded support- defined as production shocks and define the (multiplicative) transition function as production rule. In dealing with unbounded shocks, Nishimura et al. (2006) follow the line traced by Stachursky (2002), Nishimura and Stachursky (2005) and Zhang (2007).

The present paper istead assumes that shocks have bounded support. However, such assumption can be also removed, and is made only to avoid an unnecessary complication of the model. The links between our model and the relevant contribution by Mitra and Roy (2007) will be highlighted in Subsection 2.2. For a broader idea of the stochastic growth models and their fields of applications, see e.g. Snadland and McGilchrist (1979), Den Haan and Marcet (1990), Taylor and Uhlig (1990), Ausloos and Kowalski (1992), Gadomski (1996, 2003), Ausloos and Vanderwalle (1996), Gadomski and Ausloos (2006), Vanderwalle and Ausloos (1996a, 1996b, 1997).

From a technical perspective, our paper is particularly close to Grandell (1997) and Cinlar (1995). Grandell deals with the use of Mixed Poisson Processes on the line in the context of server queues, while Cinlar applies a point process perspective to model spatial queues. It is also worth mentioning the article by Cerqueti et al. (2009), which uses SMPP to model a rather complex reinsurance problem.

The remaining part of this paper is organized as follows. In Section 2 the model is presented, with the main definitions and a comparative analysis between our proposal and Mitra and Roy (2007). Section 3 contains the estimate of the stock of resource accumulated at a future date, along with the discussion of the exhaustion of R. The last Section presents our conclusions. The Appendix contains the proof of the main result and some brief notes on SMPPs.

\section{The model}

In this section the basic ingredients of our model are outlined. We proceed in two steps: in the first, we provide preliminaries and notations on the problem; in the second, a comparison of our model with that of Mitra and Roy (2007) is performed, in order to clarify our formal language and explain 
the generality of our proposal.

\subsection{Formal introduction to the problem: preliminaries and notation}

Consider a replenishable resource $\mathrm{R}$ whose available stock evolves randomly. Such a random evolution is captured by a suitable filtered probability space $\left(\Omega, \mathcal{F},\left\{\mathcal{F}_{t}\right\}_{t \geq 0}, P\right)$, where the filtration $\left\{\mathcal{F}_{t}\right\}_{t \geq 0}$ represents the available time-dependent relevant information (see Zuyev, 2006).

The evolution of the available stock of $R$ is driven by the events occurring in the environment where the resource is contextualized. Such events will occur at random times, and their effect on $R$ is not constant.

In order to proceed, we need to introduce the set $\mathcal{T}$ of the stopping times associated to the filtered probability space $\left(\Omega, \mathcal{F},\left\{\mathcal{F}_{t}\right\}_{t \geq 0}, P\right)$ :

$$
\mathcal{T}:=\left\{\tau: \Omega \rightarrow[0,+\infty] \mid\{\tau \leq t\} \in \mathcal{F}_{t}, \forall t \geq 0\right\}
$$

We assume that the sequence of events affecting $R$ can be well represented through quantitative parameters, and we model it by introducing a marked temporal process

$$
\mathbf{S}=\left\{\left(\tau_{i}, \xi_{i}\right)\right\}_{i \in \mathbb{N}},
$$

where:

- $i \in \mathbb{N}$ is the index putting in order the events, in the sense that the $i+1$-th event is the first one occurring after the $i$-th event;

- $\tau_{i} \in \mathcal{T}$ is the random time when the $i$-th event occurs;

- $\xi_{i} \in \mathcal{F}_{\tau_{i}}$ takes values in $\mathbb{R}$ and represents the entity of the $i$-th jump in the environment, i.e.: the random mark of the $i$-th event, for each $i \in \mathbb{N}$.

The couples in $\mathbf{S}$ will be denoted hereafter simply as (times and marks of) events.

A preliminary assumption summarizing some requirements we claim for $\mathbf{S}$ follows.

Assumption 1. The following conditions are true hereafter.

(1-A) The random variables $\xi_{i}$ 's are i.i.d..

(1-B) The $\xi_{i}$ 's have compact support $\left[a_{S}, b_{S}\right]$, where $a_{S}<0<b_{S}$.

(1-C) $\xi_{i}$ is independent from $\left\{\tau_{i}\right\}_{i \in \mathbb{N}}$, for each $i \in \mathbb{N}$.

Condition (1-A) is quite standard. It states that the marks of the events are not linked to one another, and they range on a unique interval.

Condition (1-B) is rather technical, and provides information on the variation range of the marks of the events. In particular, it states that the quantitative process capturing the mark of the events is bounded. This condition is not restrictive, in that it can be seen as a conventional agreement on 
the scale of measure for the marks of the events captured by $\mathbf{S}$.

Condition (1-C) formalizes the absence of a relationship between the marks of the events and the times of their occurrence. Hence, no information on the mark of the jumps may be derived by the knowledge of their time of occurrence. This assumption is quite similar to the stationarity property of the transition probabilities in the context of Markov chains.

As already stated above, the marked temporal process $\mathbf{S}$ drives the random evolution of the stock of resource. Each jump -or event- in $\mathbf{S}$ becomes a jump in a new marked temporal process $\mathbf{U}$ which represents a contribution to the aggregate stock of R. More precisely, there exists a suitable process transformation $\Phi$ such that times and marks of $\mathbf{S}$ become times and marks of $\mathbf{U}$.

It is useful to formally introduce process $\mathbf{U}$ first, and then transformation $\Phi$.

The marked temporal process $\mathbf{U}$ contains the random contributions to the stock of R:

$$
\mathbf{U}=\left\{\left(\theta_{i}, \zeta_{i}\right)\right\}_{i \in \mathbb{N}}
$$

where:

- analogously to the case of $\mathbf{S}, i \in \mathbb{N}$ provides the natural chronological order of the random contributions collected in $\mathbf{U}$;

- $\theta_{i} \in \mathcal{T}$ is the random time when the $i$-th contribution is registered;

- $\zeta_{i} \in \mathcal{F}_{\theta_{i}}$ takes values in $\mathbb{R}$ and represents the mark of the $i$-th jump contributing to the stock of $\mathrm{R}$, for each $i \in \mathbb{N}$.

The couples in $\mathbf{U}$ will be denoted hereafter simply as (times and marks of) contributions.

In our model, the sign of the marks of the contributions is relevant: $\zeta_{i}>0$ means that the $i$-th contribution augments the stock of available resource, while $\zeta_{i}<0$ means it reduces it.

We now formalize how the transformation $\Phi$ works.

Let us introduce a sequence of adjunctive i.i.d. random variables defined on $(\Omega, \mathcal{F}, P)$

$$
W=\left\{w_{i}\right\}_{i \in \mathbb{N}} \quad: \quad w_{i}(\omega) \in[0,+\infty), \forall \omega \in \Omega ; i \in \mathbb{N}
$$

and a measurable bounded transformation

$$
\phi:[0,+\infty] \times \mathbb{R} \times \Omega \rightarrow \mathbb{R} \times \Omega \quad: \quad\left(\tau_{i}, \xi_{i}\right)(\omega) \mapsto_{\phi} \phi\left(\tau_{i}, \xi_{i}\right)(\omega), \forall i \in \mathbb{N} .
$$

The transformation $\Phi$ is a function defined as follows:

$$
\Phi:[0,+\infty] \times \mathbb{R} \times[0,+\infty) \times \Omega \rightarrow[0,+\infty] \times \mathbb{R} \times \Omega
$$

such that

$$
\Phi\left(\tau_{i}, \xi_{i}, w_{i}\right)(\omega):=\left(\tau_{i}+w_{i}, \phi\left(\tau_{i}, \xi_{i}\right)\right)(\omega), \quad \forall i \in \mathbb{N} .
$$


By defining

$$
\theta_{i}(\omega):=\tau_{i}(\omega)+w_{i}(\omega), \quad \zeta_{i}(\omega):=\phi\left(\tau_{i}, \xi_{i}\right)(\omega) \quad \forall i \in \mathbb{N}, \omega \in \Omega
$$

we have a representation of the contributions of the process $\mathbf{U}$ associated to the events of the process S.

The term $w_{i}$ formalizes that the propagation of the $i$-th event to the related $i$-th contribution may be randomly delayed rather than instantaneous. The limiting case of $w_{1}^{(i)}(\omega)=0$ means that the $i$-th event is translated instantaneously in the $i$-th contribution, for $\omega \in \Omega$.

Function $\phi$ explains the relationship between the mark of the contributions and the mark of the related events. Such a link is twofold: firstly, the mark of each event drives the mark of the corresponding jump in the process $\mathbf{U}$; secondly, the same event may generate two contributions with different marks when it occurs in two different epochs, and such an evidence is captured by the dependence of $\zeta_{i}$ also on $\tau_{i}$, for each $i$.

Remark 1. Since function $\phi$ is assumed to be bounded, then the random marks of the contributions have bounded support, and we can assume that such a support is contained in $\left[a_{U}, b_{U}\right]$, with $a_{U}<$ $0<b_{U}$.

To model consumption -which occurs deterministically in time- we adopt a discrete-time approach. For this purpose, we introduce a sequence of deterministic times $\left\{t_{h}\right\}_{h \in \mathbb{N}} \in[0,+\infty)$ such that $t_{0}=0$ and $t_{h}<t_{h+1}$, and a sequence of deterministic percentages $\left\{\alpha_{h}\right\}_{h \in \mathbb{N}} \in[0,1]$. For each $h$, the percentage of resource consumed at time $t_{h}$ is $\alpha_{h}$.

We are now able to introduce the definition of the random quantity analyzed in the paper.

Definition 1. The stock of $\mathrm{R}$ available at time $t>0$ is a random variable defined as the aggregation of the marks of the contributions of $\mathbf{U}$ minus consumption up to time $t$, and it depends also on the deterministic initial amount $y$ of $\mathrm{R}$.

For the sake of simplicity, we first define the stochastic process of interest, and then provide the complete definition of the available stock of resource with consumption.

We introduce the stochastic process $\left\{N_{t}^{(y)}\right\}_{t \geq 0}$ as follows:

$$
N_{t}^{(y)}(\omega)=N_{0}^{(y)}+\sum_{i=1}^{+\infty} \zeta_{i}(\omega) \mathbf{1}_{\left\{\theta_{i}(\omega) \leq t\right\}}, \quad \forall \omega \in \Omega
$$

with $N_{0}^{(y)}=y$. By subtracting consumption, we obtain the final formulation for the stock of available resource as follows:

$$
R_{t}^{(y)}(\omega)=\left[N_{t}^{(y)}(\omega)-\sum_{h=1}^{+\infty} \alpha_{h} N_{t_{h}}^{(y)}(\omega) \mathbf{1}_{\left\{t_{h} \leq t\right\}}\right]^{+},
$$

where $[\bullet]^{+}=\max \{\bullet, 0\}$.

In this work, we deal with the analysis of the depletion of $\mathrm{R}$.

Definition 2. The resource $\mathrm{R}$ with initial endowment $y>0$ is depleted (or exhausted) at time $t>0$ when $R_{t}^{(y)}(\omega)=0$, for each $\omega \in \Omega$. 0 is the state of depletion. 
Remark 2. In general, the definition of the marks $\xi$ 's through function $\phi$ in (8) does not impose to treat 0 as an absorbing state for the dynamics of the available stock of $\mathrm{R}$. Indeed, an event can generate a contribution with positive mark, even if the resource was previously exhausted.

Exhaustion can be viewed from two different perspectives. Specifically, it can be associated to a time or to a (negative) critical mark of contribution such that the aggregate amount of the resource becomes null. We analyze both these different points of view.

The following definition collects some important concepts regarding depletion when time is considered.

Definition 3. The first time of depletion $\theta_{y} \in \mathcal{T}$ is the first exit time from above of the dynamic $R_{t}^{(y)}$ from the barrier 0 , i.e.:

$$
\theta_{y}(\omega)=\inf \left\{t>0: R_{t}^{(y)}(\omega)=0\right\},
$$

for each $\omega \in \Omega$, with the convention that $\inf \emptyset=+\infty$.

The resource $\mathrm{R}$ with initial endowment $y>0$ goes exhausted almost surely when

$$
P\left(\left\{\omega \in \Omega: \theta_{y}(\omega)=+\infty\right\}\right)=0 .
$$

$\mathrm{R}$ goes exhausted almost surely within a time horizon $T>0$ when

$$
P\left(\left\{\omega \in \Omega: \theta_{y}(\omega) \leq T\right\}\right)=1
$$

For sake of completeness, we now reinterpret depletion by introducing the critical mark of the contribution which leads to null aggregate amount of the resource.

Fix $y>0$ and $i \in \mathbb{N}$. Since the marks of the contributions are bounded (see Remark 1) there exists a critical random mark $\Theta_{i}^{(y)}$ with support in $(-\infty, 0)$ such that: if $\zeta_{i}=\Theta_{i}^{(y)}$, then

$$
R_{t}^{(y)}(\omega)=0, \quad \forall \omega \in \Omega: \theta_{i}(\omega) \leq t<\theta_{i+1}(\omega)
$$

The family $\left\{\Theta_{i}^{(y)}\right\}_{i \in \mathbb{N}}$ represents the set of killing jumps, which are responsible of the exhaustion of the resource. In this respect, the concepts of depletion introduced in Definition 3 can be rewritten by means of the thresholds $\Theta$ 's.

Definition 4. The first time of depletion $\theta_{y} \in \mathcal{T}$ can be defined as follows:

$$
\theta_{y}(\omega)=\inf _{i \in \mathbb{N}}\left\{\theta_{i}(\omega): \zeta_{i}(\omega)=\Theta_{i}^{(y)}(\omega)\right\}
$$

for each $\omega \in \Omega$, with the convention that $\inf \emptyset=+\infty$.

The resource $\mathrm{R}$ with initial endowment $y>0$ goes exhausted almost surely when

$$
\max _{i \in \mathbb{N}} P\left(\left\{\omega \in \Omega: \zeta_{i}(\omega)=\Theta_{i}^{(y)}(\omega)\right\}\right)=1
$$

$\mathrm{R}$ goes exhausted almost surely within a time horizon $T>0$ when

$$
\max _{i \in \mathbb{N}} P\left(\left\{\omega \in \Omega: \theta_{i}(\omega) \leq T\right\} \cap\left\{\omega \in \Omega: \zeta_{i}(\omega)=\Theta_{i}^{(y)}(\omega)\right\}\right)=1 .
$$




\subsection{Discussion on the model}

The purpose of this section is to point out the generality of our model. To do this, we provide a comparative analysis of the proposed framework with the stochastic growth model of Mitra and Roy (2007), which is the most recent relevant contribution to the field of stochastic growth applied to the evolution of the resources. For the sake of clarity, when possible we adapt the notation of the quoted paper to that of the present study.

In Mitra and Roy (2007) the stock of resource is a stochastic process $\left\{R_{t}^{(y)}\right\}_{t \in \mathbb{N}}$ with nonnegative support, where $y>0$ is the stock of resource at time 0 . The evolution of the stock is affected by random shocks $\left\{r_{t}\right\}_{t \in \mathbb{N}}$, which are i.i.d. random variables with support $\left[a_{S}, b_{S}\right]$, measured at deterministic times $t \in \mathbb{N}$. Such an evolution is driven by a predetermined transition function $G:[0,+\infty) \times\left[a_{S}, b_{S}\right] \rightarrow[0,+\infty)$ such that $R_{t+1}^{(y)}=G\left(R_{t}^{(y)}, r_{t}\right)$, which satisfies three Assumptions:

$(M R 1) G(0, r)=0$, for each $r \in\left[a_{S}, b_{S}\right]$

$(M R 2) \lim _{R \rightarrow+\infty} \frac{G\left(R, b_{S}\right)}{R}=\rho<1 ;$

$(M R 3) G$ is nondecreasing in $r$.

$(M R 4)$ For any $R>0, P\left(r \in\left[a_{S}, b_{S}\right]: G(R, r)>G\left(R, a_{S}\right)\right)=1$. Furthermore, for each $R^{\prime}<R^{\prime \prime}$, one has:

$$
\lim _{r \rightarrow a_{S}} \sup _{R \in\left[R^{\prime}, R^{\prime \prime}\right]}\left[G(R, r)-G\left(R, a_{S}\right)\right]=0
$$

Mitra and Roy (2007)'s framework is contained in our general proposal. Rather than in a deterministic time set up, the random shocks $\mathbf{S}$ in (2) are here modeled through a marked temporal process, with marks $\xi$ 's and random times $\tau$ 's. According to Mitra and Roy, the marks $\xi$ 's are i.i.d. (see Assumption 1-A). The transition function $\Phi$ in (7) concurs with formula (10) to the construction of the evolution of the stock of resource. The dependence of the marks $\zeta$ 's of the process $\mathbf{U}$ on the random times $\tau$ 's (see formula (8)) allows us to formalize a non-Markovian transition function which depends also on time. In this respect, we include and improve Mitra and Roy's set up. We do not need Assumption $(M R 1)$, and 0 is not an absorbing state in our framework. We relax $(M R 3)$, in that the effect of a shock is biased by the random time of its occurrence in our model. Further, we do not need to state a threshold for the maximum sustainable level of resource. Hence Assumption $(M R 2)$ is not stated in our theoretical setting. Lastly, Assumption (MR4) is a technicality of Mitra and Roy's framework which is unnecessary in our model.

\section{The problem}

We are now able to introduce the basic problem of the present research, that in plain words can be illustrated as follows: 
we want to provide an estimation of the expected value of the variable $R_{t}^{(y)}$, in order to derive insights on the occurrence of the depletion of $\mathrm{R}$ within a fixed time interval $[\underline{T}, \bar{T}] \subseteq[0,+\infty)$.

In order to deal with our problem, a key requirement on the process $\mathbf{S}$ is needed.

Assumption 2. S is a Spatial Mixed Poisson Process (SMPP) with mixing distribution $\Pi$ and baseline intensity measure $M$.

For reader convenience, we report the formal definition of an SMPP with some significant results in the Appendix.

Assumption 2 plays a relevant role. We develop some arguments explaining why $\mathbf{S}$ must fulfil it.

In our problem we deal with an estimation of the expected stock of $\mathrm{R}$ available at time $t$. Hence, we have to focus on $R_{t}^{(y)}$ as written in formula (10). Therefore, we need to know the stochastic structure of the marked temporal process $\mathbf{U}$. Now, the contributions in $\mathbf{U}$ are defined through a transformation of the events in $\mathbf{S}$, as formulas (3) and (8) assure. This implies that the information regarding $\mathbf{U}$ must be derived by the definitions of the process $\mathbf{S}$ in (2) and of the transformation $\Phi$ in (7). In particular, transformation $\Phi$ is such that the mark $\zeta_{i}$ depends on the couple $\left(\tau_{i}, \xi_{i}\right)$, for each $i \in \mathbb{N}$. The dependence between the $\zeta$ 's and the process $\mathbf{S}$ imposes to abandon the familiar simple model where $\mathbf{S}$ is a one-dimensional Mixed Poisson Process, and rather assume that $\mathbf{S}$ is a SMPP. Indeed, only in this case we can apply a mathematical result, stating that if $\mathbf{S}$ is a SMPP and $\Phi$ is defined as in (7), then $\mathbf{U}$ is a SMPP as well (see Theorem 1 in the Appendix). This invariance property is crucial in our estimation procedure.

To proceed, consider $0<r_{1}<r_{2}<t, \rho>0$ and introduce the time intervals

$$
I=\left[r_{1}, r_{2}\right], \quad J_{t}^{(\rho)}=[t, t+\rho) .
$$

The intervals $I$ and $J_{t}^{(\rho)}$ are the test and the investigated set, respectively, and constitute the basis of our estimation procedure.

We now define the regions $\mathcal{I}_{S} \equiv I \times\left[a_{S}, b_{S}\right], \mathcal{I}_{U} \equiv I \times\left[a_{U}, b_{U}\right]$ and $\mathcal{J}_{t}^{(\rho)} \equiv J_{t}^{(\rho)} \times\left[a_{U}, b_{U}\right]$.

With a reasonable use of improper notations, we refer to $\mathbf{S}(D)$ and $\mathbf{U}(D)$ as the number of the elements of the Spatial Point Process $\mathbf{S}$ and $\mathbf{U}$, respectively, that are contained in $D \subseteq \mathbb{R}^{2}$.

Remark 3. Since a SMPP is a simple process (see Appendix, Lemma 1) and regions $\mathcal{I}_{S}$ and $\mathcal{I}_{U}$ are bounded, then $\mathbb{E}\left[\mathbf{S}\left(\mathcal{I}_{S}\right)\right]<+\infty, \mathbb{E}\left[\mathbf{U}\left(\mathcal{I}_{U}\right)\right]<+\infty$.

We provide an estimate of the available amount of $\mathrm{R}$ within $J_{t}^{(\rho)}$. Then, by setting a limit for $\rho \rightarrow 0^{+}$, an estimation of the stock of resorce available at time $t$ will be found. The procedure moves from the knowledge of the following quantities:

- the number $\mathbf{S}\left(\mathcal{I}_{S}\right)$ of the events occurring during $I$;

- the number $\mathbf{U}\left(\mathcal{I}_{U}\right)$ of the contributions registered during $I$; 
- the number of events occurred over $I$ and delayed as contributions to $\mathrm{R}$ in the same time interval. We denote this quantity as $\mathbf{U}_{\left(\mathcal{I}_{S}\right)}\left(\mathcal{I}_{U}\right)$.

We now consider a partition of the interval $\left[a_{U}, b_{U}\right]$ as follows:

$$
\left[a_{U}, b_{U}\right] \equiv\left\{\bigcup_{s=1}^{j-1}\left[c_{s-1}^{(j)}, c_{s}^{(j)}\right)\right\} \cup\left[c_{j-1}^{(j)}, c_{j}^{(j)}\right], \quad j \in \mathbb{N},
$$

where the terms of the partition satisfies the following conditions:

$$
\left\{\begin{array}{c}
a_{U}=c_{0}^{(j)}<c_{1}^{(j)}<\cdots<c_{j}^{(j)}=b_{U} \\
\lim _{j \rightarrow+\infty} \max _{s \in\{1, \ldots j\}}\left(c_{s}^{(j)}-c_{s-1}^{(j)}\right)=0 .
\end{array}\right.
$$

Moreover, we consider a partition of the time interval $J_{t}^{(\rho)}$ as follows:

$$
J_{t}^{(\rho)} \equiv\left\{\bigcup_{r=1}^{j-1}\left[u_{r-1}^{(j)}, u_{r}^{(j)}\right)\right\} \cup\left[u_{j-1}^{(j)}, u_{j}^{(j)}\right], \quad j \in \mathbb{N},
$$

where the terms of the partition satisfies the following conditions:

$$
\left\{\begin{array}{c}
t=u_{0}^{(j)}<u_{1}^{(j)}<\cdots<u_{j}^{(j)}=t+\rho ; \\
\lim _{j \rightarrow+\infty} \max _{r \in\{1, \ldots j\}}\left(u_{r}^{(j)}-u_{r-1}^{(j)}\right)=0 .
\end{array}\right.
$$

For the sake of simplicity, the generic term of the partition of $J_{t}^{(\rho)}$ will be denoted as $T_{r}^{(j)}$.

Then $\mathcal{J}_{t}^{(\rho)}$ can be written as:

$$
\mathcal{J}_{t}^{(\rho)} \equiv \bigcup_{r, s=1}^{j} \Delta_{r, s}^{(j)}
$$

where

$$
\Delta_{r, s}^{(j)} \equiv \begin{cases}T_{r}^{(j)} \times\left[c_{s-1}^{(j)}, c_{s}^{(j)}\right), & \text { for } s \leq j-1 \\ T_{r}^{(j)} \times\left[c_{s-1}^{(j)}, c_{s}^{(j)}\right], & \text { for } s=j .\end{cases}
$$

for each $j \in \mathbb{N}$ and $r \leq j$.

It is worth noting that $\Delta_{r, s}^{(j)}$ defined in (20) depends on $h$ and $t$. However, to avoid an unnecessarily cumbersome notation, we omit an explicit reference to such a dependence.

The event $A$ driving our estimate can be formalized as follows:

$$
A=\left\{\mathbf{S}\left(\mathcal{I}_{S}\right)=m_{S}\right\} \cap\left\{\mathbf{U}\left(\mathcal{I}_{U}\right)=m_{U}\right\} \cap\left\{\mathbf{U}_{\left(\mathcal{I}_{S}\right)}\left(\mathcal{I}_{U}\right)=m_{S, U}\right\},
$$

where $m_{S}, m_{U}, m_{S, U} \in \mathbb{N}$.

Remark 4. We can propose two perspectives to interpret the practical significance of conditioning with respect to $A$. On the one hand, we can assume that the test interval I is fully contained in the past. This means that "today" is a date between $r_{2}$ and $t$. In this case, we are aware about the realizations of the random variables $\mathbf{S}\left(\mathcal{I}_{S}\right), \mathbf{U}\left(\mathcal{I}_{U}\right)$ and $\mathbf{U}_{\left(\mathcal{I}_{S}\right)}\left(\mathcal{I}_{U}\right)$, and our computations make sense. On the other hand, one can provide the estimation on the basis of a hypothetical value of the realizations of the quantities variables $\mathbf{S}\left(\mathcal{I}_{S}\right), \mathbf{U}\left(\mathcal{I}_{U}\right)$ and $\mathbf{U}_{\left(\mathcal{I}_{S}\right)}\left(\mathcal{I}_{U}\right)$. In doing this, we are able to derive information on the expected value of the quantity of resource at a given time on the basis of its past evolution. 
We fix $j \in \mathbb{N}$ and $r, s \in\{1, \ldots, j\}$ and denote by $b_{r, s}^{(j)}$ the conditional expectation of the number of the contributions in $\Delta_{r, s}^{(j)}$, given the occurrence of the event $A$ :

$$
b_{r, s}^{(j)} \equiv \mathbb{E}\left[\mathbf{U}\left(\Delta_{r, s}^{(j)}\right) \mid A\right] .
$$

The next result provides an explicit formula to compute $b_{r, s}^{(j)}$, for any $j \in \mathbb{N}$ and $r, s \in\{1, \ldots, j\}$.

\section{Proposition 1.}

$$
b_{r, s}^{(j)}=\sum_{n=0}^{+\infty} \sum_{l=0}^{n} n \cdot \Gamma_{1}(n, l) \cdot \Gamma_{2}(n, l) \cdot \Gamma_{3}(n, l) \cdot \Gamma_{4}(n, l),
$$

with

$$
\left\{\begin{array}{l}
\Gamma_{1}(n, l)=\frac{\left[M_{\left(\mathcal{I}_{S}\right)}\left(\Delta_{r, s}^{(j)}\right)\right]^{n-l}}{(n-l) !} \\
\Gamma_{2}(n, l)=\frac{\left[M_{\left(\mathcal{I}_{S}\right)}\left(\Delta_{r, s}^{(j)}\right)\right]^{l}}{l !} \\
\Gamma_{3}(n, l)=\int_{0}^{+\infty} \lambda^{n-l} e^{-\lambda M_{\left(\mathcal{I}_{S}\right)}\left(\Delta_{r, s}^{(j)}\right)} u\left(\lambda ; \mathcal{I}_{S}, \mathcal{I}_{U}, m_{S}, m_{U}, m_{S, U}\right) \mathrm{d} \lambda \\
\Gamma_{4}(n, l)=\int_{0}^{+\infty} \lambda^{l} e^{-\lambda M_{\left(\mathcal{I}_{S}\right)}\left(\Delta_{r, s}^{(j)}\right)} u\left(\lambda ; \mathcal{I}_{S}, \mathcal{I}_{T}, m_{S}, m_{U}, m_{S, U}\right) \mathrm{d} \lambda .
\end{array}\right.
$$

where $M_{\left(\mathcal{I}_{S}\right)}$ is the baseline intensity measure of the $\operatorname{SMPP} \mathbf{U}_{\left(\mathcal{I}_{S}\right)}$ (see Lemma 2 in the Appendix) and

$$
u\left(\lambda ; \mathcal{I}_{S}, \mathcal{I}_{U}, m_{S}, m_{U}, m_{S, U}\right)=\frac{\lambda^{m_{U}-m_{S, U}+m_{S}} e^{-\lambda\left[M_{\left(\mathcal{I}_{S}\right)}\left(\mathcal{I}_{U}\right)+M\left(\mathcal{I}_{S}\right)\right]} u(\lambda)}{\int_{0}^{\infty} \lambda^{m_{U}-m_{S, U}+m_{S}} e^{-\lambda\left[M_{\left(\mathcal{I}_{S}\right)}\left(\mathcal{I}_{U}\right)+M\left(\mathcal{I}_{S}\right)\right]} u(\lambda) d \lambda}
$$

is the distribution of $\lambda$ (see Eq. (35)).

The proof is reported in the Appendix.

Proposition 1 allows to get an upper and a lower approximation of $\mathbb{E}\left[R_{t}^{y} \mid A\right]$, which is the expected stock of resource available at time $t>0$ under the conditioning of the event $A$.

Consider the following quantities:

$$
\begin{aligned}
& \bar{\phi}_{j}=\left[\lim _{\rho \rightarrow 0^{+}}\left\{\sum_{r=1}^{j} \sum_{s=1}^{j}\left[1-\sharp\left(T_{r}^{(j)}\right) \hat{\alpha}\left(T_{r}^{(j)}\right)\right] \cdot c_{s}^{(j)} b_{r, s}^{(j)}\right\}\right]^{+}, \\
& \hat{\phi}_{j}=\left[\lim _{\rho \rightarrow 0^{+}}\left\{\sum_{r=1}^{j} \sum_{s=1}^{j}\left[1-\sharp\left(T_{r}^{(j)}\right) \bar{\alpha}\left(T_{r}^{(j)}\right)\right] \cdot c_{s-1}^{(j)} b_{r, s}^{(j)}\right\}\right]^{+},
\end{aligned}
$$

where

$$
\sharp\left(T_{r}^{(j)}\right)=\left|\left\{h \in \mathbb{N} \mid t_{h} \in T_{r}^{(j)}\right\}\right|, \quad \hat{\alpha}\left(T_{r}^{(j)}\right)=\max _{h \mid t_{h} \in T_{r}^{(j)}}\left\{\alpha_{h}\right\}, \quad \bar{\alpha}\left(T_{r}^{(j)}\right)=\min _{h \mid t_{h} \in T_{r}^{(j)}}\left\{\alpha_{h}\right\} .
$$

For any $j \in \mathbb{N}$, we have

$$
\hat{\phi}_{j} \leq \mathbb{E}\left[R_{t}^{(y)} \mid A\right] \leq \bar{\phi}_{j}
$$

By definition, $\left\{\hat{\phi}_{j}\right\}$ is non-decreasing and $\left\{\bar{\phi}_{j}\right\}$ is non-increasing with respect to $j$. Moreover, there exists a time-dependent quantity $p(t)$ such that

$$
\lim _{j \rightarrow+\infty} \hat{\phi}_{j}=\lim _{j \rightarrow+\infty} \bar{\phi}_{j}=p(t) .
$$


Taking the limit as $j \rightarrow+\infty$, by (25) and (26) we obtain that $p(t)$ represents the estimate of the stock of $\mathrm{R}$ available at time $t$ with initial endowment $y$ given the information collected in $A$, i.e.:

$$
\mathbb{E}\left[R_{t}^{y} \mid A\right]=p(t) .
$$

Starting from (27), we argue that the resource will deplete in time interval $[\underline{T}, \bar{T}]$ if and only if there exists $t^{\star} \in[\underline{T}, \bar{T}]$ such that

$$
t^{\star}=\inf \{t>0 \mid p(t)=0\} .
$$

Formally, we can also write the probability that the resource goes exhausted in $[\underline{T}, \bar{T}]$ as $P\left(\left\{\omega \in \Omega: \theta_{y}(\omega) \leq t^{\star}\right\}\right)$, where $\theta_{y}$ and $t^{\star} \in[\underline{T}, \bar{T}]$ are defined as in (11) and (28), respectively.

\section{Conclusions}

This paper concerns the exhaustion of a replenishable resource, which is a very important problem in the field of environmental research. To pursue our scope, we provide a theoretical estimate of the available stock of $\mathrm{R}$ at a fixed time. In dealing with a SMPP framework for the stock of resource, we extend and improve the relevant Markovian stochastic growth models already present in the literature.

Moreover, the lack of independence between the marks of the contributions and times and marks of the events (see the definition of function $\phi$ in Formula (8)) imposes us to abandon the usual Mixed Poisson framework and to treat the process of the events as a spatial process. In fact, consider the familiar simple model described as follows:

(i) the times of the events are described by a one-dimensional Mixed Poisson Process;

(ii) the marks of the events are i.i.d. and independent of the arrival times;

(iii) the marks of the contributions are i.i.d. and independent from the process $\mathbf{S}$.

Then, the process $\mathbf{U}$ has the same stochastic structure described by (i) and (ii). Generally this is no longer true when condition (iii) fails. As we have argued, it is instead the stochastic structure of SMPP that remains invariant (see Theorem 1 in the Appendix), even in the case of stochastic dependence between the marks of the contributions and the process $\mathbf{S}$.

Our theoretical model includes several aspects of significant real-world examples. In this respect, the paradigmatic cases of water and oil have been presented in some detail. In doing this, we have provided a justification of the constitutive hypotheses of the theoretical model and an identification of the scientific ground of the research. However, it could be interesting to perform a purely econometric analysis of a SMPP, to obtain empirically-driven insights on frequencies and marks of the jumps. This is a challenging issue and, to the best of our knowledge, has been neglected in the literature. We are currently working in this direction. 


\section{References}

[1] Anderson, M.L., Mierzwa, M.D., Kavvas, M.L., 2000. Probabilistic seasonal forecasts of droughts with a smplified coupled hydrologic-atmospheric molde for water resource planning. Stochastic Environmental Research and Risk Assessment 14, 263-274.

[2] Athreya, K.B., 2003. Stationary measures for some Markov chain models in ecology and economics. Economic Theory 23, 107-122.

[3] Ausloos, M., Kowalski, J.M., 1992. Stochastic models of two-dimensional fracture. Physical Review B 45(22), 12830.

[4] Ausloos, M., Vanderwalle, N., 1996. Growth models with internal competition. Acta Physica Polonica Series B 27, 737-746.

[5] Cinlar, E., 1995. An introduction to spatial queues. In: Advances in queueing, Probability and Stochastics Series, CRC, Boca Raton, 103-118.

[6] Cerqueti, R., Foschi, R., Spizzichino, F., 2009. A Spatial Mixed Poisson Framework for Combination of Excess-of-Loss and Proportional Reinsurance Contracts. Insurance: Mathematics and Economics, 45(1), 59-64.

[7] Chen, C., Huang, G.H., Li, Y.P., Zhou, Y., 2013. A robust risk analysis method for water resources allocation under uncertainty. Stochastic Environmental Research and Risk Assessment $27,713-723$.

[8] Dai, X., Li, Z., Lin, S., Xu, W., 2012. Assessment and zoning of eco-environmental sensitivity for a typical developing province in China. Stochastic Environmental Research and Risk Assessment 26, 1095-1107.

[9] Daley, D.J., Vere-Jones, D., 1988. An Introduction to the Theory of Point Processes. New York: Springer-Verlag.

[10] Dearbor, D.C., Kark, S., 2010. Motivations for conserving urban biodiversity. Conservation Biology 24, 432440 .

[11] Den Haan, W.J., Marcet, A., 1990. Solving the stochastic growth model by parameterizing expectations. Journal of Business \& Economic Statistics 8(1), 31-34.

[12] Foschi, R., Spizzichino, F., 2008. The role of the Order Statistic Property in Mixed Spatial Poisson Processes. In: Proceedings of the International Workshop on Applied Probability. Université de Technologie de Compiégne. 
[13] Gadomski, A., 1996. Stochastic approach to the evolution of some polycrystalline (bio) polymeric complex systems. Chemical physics letters 258(1), 6-12.

[14] Gadomski, A., 2003. Multilineal Random Patterns Evolving Subdiffusively in Square Lattice. Fractals 11(01), 233-241.

[15] Gadomski, A., Ausloos, M., 2006. Agglomeration/Aggregation and Chaotic Behaviour in dDimensional Spatio-Temporal Matter Rearrangements Number-Theoretic Aspects. In: The Logistic Map and the Route to Chaos, Springer-Verlag, 275-294.

[16] Grandell, J., 1997. Mixed Poisson Processes. Chapman \& Hall, London.

[17] Gu, J.J., Guo, P., Huang, G.H., Shen, N., 2013. Optimization of the industrial structure facing sustainable development in resource-based city subjected to water resources under uncertainty. Stochastic Environmental Research and Risk Assessment 27, 659-673.

[18] Guo, P., Huang, G.H., 2009. Two-stage fuzzy chance-constrained programming: application to water resources management under dual Stochastic Environmental Research and Risk Assessment 23, 349-359.

[19] Herrera-Silveira, J.A., Morales-Ojeda, S.M. 2009. Evaluation of the health status of a coastal ecosystem in southeast Mexico: assessment of water quality, phytoplankton and submerged aquatic vegetation. Marine Pollution Bulletin 59, 72-86.

[20] Hoppe, H., Weilandt, M., Orth, H., 2004. A combined water management approach based on river water quality standards. Journal of Environmental Informatics 3(2), 67-76.

[21] Hu, Q., Huang, G.H., Liu, Z., Fan, Y., Li, L., 2012 Inexact fuzzy two-stage programming for water resources management in an environment of fuzziness and randomness. Stochastic Environmental Research and Risk Assessment 26, 261-280.

[22] Jones, J.A., Post, D.A., 2004. Seasonal and successional streamflow response to forest cutting and regrowth in the northwest and eastern United States. Water Resources Research 40, 0520310520319 .

[23] Li, Y., Zhua, X., Sun, X., Wang, F., 2010. Landscape effects of environmental impact on bayarea wetlands under rapid urban expansion and development policy: a case study of Lianyungang, China. Landscape and Urban Planning 94, 218-227.

[24] Li, Y.P., Huang, G.H., Nie, S.L. 2006. An interval-parameter multi-stage stochastic programming model for water resources management under uncertainty. Advances in Water Resources 29, 776-789. 
[25] Loukas, A., Vasiliades, L., Dalezios, N.R., 2002. Potential climate change impacts on flood producing mechanisms in southern British Columbia, Canada using the CGCMA1 simulation results. Journal of Hydrology 259, 163-188.

[26] Ludwig, D., 1999. Is it meaningful to estimate a probability of extinction?. Ecology 80, 298-310.

[27] Lv, Y., Huang, G.H., Li, Y.P., Sun, W., 2012. Managing water resources system in a mixed inexact environment using superiority and inferiority measures. Stochastic Environmental Research and Risk Assessment 26, 681-693.

[28] Maqsood, I., Huang, G.H., Yeomans, J.S., 2005. An interval-parameter fuzzy two-stage stochastic program for water resources management under uncertainty. European Journal of Operational Research 167, 208-225.

[29] Mitra, T., Roy, S., 2007. On the possibility of extinction in a class of Markov processes in economics. Journal of Mathematical Economics 43, 842-854.

[30] Nishimura, K., Rudnicki, R., Stachurski, J., 2006. Stochastic optimal growth with nonconvexities. Journal of Mathematical Economics 42, 74-92.

[31] Nishimura, K., Stachurski, J., 2005. Stability of stochastic optimal growth models: a new approach. Journal of Economic Theory 122, 100-118.

[32] Ouyang, Z.Y., Wang, X.K., Miao, H., 2000. Chinas eco-environmental sensitivity and its spatial heterogeneity. Acta Ecologica Sinica 20, 9-11.

[33] Sandland, R.L., McGilchrist, C.A., 1979. Stochastic growth curve analysis. Biometrics 255-271.

[34] Stachurski, J., 2002. Stochastic optimal growth with unbounded shock. Journal of Economic Theory 106, 40-65.

[35] Stoyan, D., Kendall, W.S., Mecke, J., 1995. Stochastic Geometry and its Applications. Chichester: John Wiley \& Sons.

[36] Sullivan, C.A., 2011. Quantifying water vulnerability: a multi-dimensional approach. Stochastic Environmental Research and Risk Assessment 25, 627-640.

[37] Tamerius, J.T., Wise, E.K., Uejio, C.K., McCoy, A.L., Comrie, A.C., 2006. Climate and human health: synthesizing environmental complexity and uncertainty. Stochastic Environmental Research and Risk Assessment 21(5), 601-613.

[38] Taylor, J.B., Uhlig, H., 1990. Solving nonlinear stochastic growth models: A comparison of alternative solution methods. Journal of Business $\& 3$ Economic Statistics 8(1), 1-17. 
[39] Tu, X., Zhang, Q., Singh, V.P., Chen, X., Liu, C.-L., Wang, S.-B., 2012. Spacetime changes in hydrological processes in response to human activities and climatic change in the south China. Stochastic Environmental Research and Risk Assessment 26, 823-834.

[40] Vanderwalle, N., Ausloos, M., 1996a. The screening of species in a Darwinistic tree-like model of evolution. Physica D: Nonlinear Phenomena 90(3), 262-270.

[41] Vanderwalle, N., Ausloos, M., 1996b. Growth of Cayley and diluted Cayley trees with two kinds of entities. Journal of Physics A: Mathematical and General 29(22), 7089.

[42] Vanderwalle, N., Ausloos, M., 1997. Construction and properties of fractal trees with tunable dimension: The interplay of geometry and physics. Physical Review E 55(1), 94.

[43] Wang, H.J., Yang, Z.S., Saito, Y., Liu, J.P., Sun, X.X., 2006. Interannual and seasonal variation of the Huanghe (Yellow River) water discharge over the past 50 years: connections to impacts from ENSO events and dams. Global and Planetary Change 50, 212-225.

[44] Yu, H.-L., Yang, S.-J., Yen, H.-J., Christakos, G., 2011. A spatiotemporal climate-based model of early dengue fever warning in southern Taiwan. Stochastic Environmental Research and Risk Assessment 25(4), 485-494.

[45] Zhang, Y., 2007. Stochastic optimal growth with a non-compact state space. Journal of Mathematical Economics 43, 115-129.

[46] Zhang, Q., Xu, C.-Y., Tao, H., Jiang, T., Chen, Y.D., 2010. Climate changes and their impacts on water resources in the arid regions: a case study of the Tarim River basin, China. Stochastic Environmental Research and Risk Assessment 24(3), 349-358.

[47] Zuyev, S., 2006. Strong Markov property of Poisson processes and Slivnyak formula. Lect. Notes in Stat., 185, 77-84.

\section{Appendix}

\section{Some notes on Spatial Mixed Poisson Processes}

In this Appendix we collect the definition and some recent results on SMPPs, which are particular marked temporal processes. For a complete treatment of stochastic processes of spatial type, see Daley and Vere-Jones (1988) and Stoyan et al. (1995).

Consider a measure space $\left(\mathbb{R}^{k}, \mathcal{B}\left(\mathbb{R}^{k}\right), M\right)$, where $\mathcal{B}\left(\mathbb{R}^{k}\right)$ is the Borel $\sigma$-algebra and $M$ is a measure which is absolutely continuous with respect to the Lebesgue measure. We also introduce a nonnegative random variable $\lambda$ with probability distribution $\Pi:[0,+\infty) \rightarrow[0,1]$. 
Definition 5. A spatial process $\mathbf{S}$ is Mixed Poisson (SMPP) with mixing distribution $\Pi$ and baseline intensity measure $M(\cdot)$ when, for $\mathcal{I} \in \mathcal{B}\left(\mathbb{R}^{k}\right)$ and for $n \in \mathbb{N}$,

$$
P(\mathbf{S}(\mathcal{I})=n)=\int_{0}^{\infty} e^{-\lambda M(\mathcal{I})} \frac{[\lambda M(\mathcal{I})]^{n}}{n !} d \Pi(\lambda) .
$$

Since $M$ is absolutely continuous with respect to the Lebesgue measure, we have the following result.

Lemma 1. A SMPP is a simple marked temporal process.

Now, assume that the SMPP $\mathbf{S}$ is such that $\mathbf{S} \equiv\left\{X_{i}\right\}_{i \in \mathbb{N}}$, where $X_{i}$ has support $\mathcal{X} \subseteq \mathbb{R}^{k}, k \in \mathbb{N}$, for any $i \in \mathbb{N}$.

Consider a sequence of i.i.d. random variables $W=\left\{w_{i}\right\}_{i \in \mathbb{N}}$ taking values on a set $\mathcal{W} \subseteq \mathbb{R}^{n}$ for some $n \in \mathbb{N}$.

Consider $\mathcal{Y} \subseteq \mathbb{R}^{k}$ and define a transformation

$$
\Phi: \mathcal{X} \times \mathcal{W} \times \Omega \rightarrow \mathcal{Y} \times \Omega
$$

where $\Phi(\cdot, w)(\omega): \mathcal{X} \rightarrow \mathcal{Y}$ is measurable for any fixed $w \in \mathcal{W}$ and $\omega \in \Omega$.

We define the transformed marked temporal process as follows:

$$
\mathbf{U}=\left\{\Phi\left(X_{i}, w_{i}\right)\right\}_{i \in \mathbb{N}}
$$

We can also write $\mathbf{U}=\Phi_{\phi}(\mathbf{S}, W)$ instead of (30).

The following theorem is particular important in our setting (see Cinlar 1995, Foschi and Spizzichino, 2008).

Theorem 1. Let $\mathbf{S}$ be a SMPP with mixing distribution $\Pi$ and baseline intensity measure $M$. Consider a sequence of i.i.d. random variables $W=\left\{w_{i}\right\}_{i \in \mathbb{N}}$, with distribution $G$ and independent of $\mathbf{S}$.

Then $\mathbf{U}=\Phi_{\phi}(\mathbf{S}, W)$ is a SMPP with the same mixing distribution $\Pi$ and intensity measure

$$
M^{*}(\mathcal{J})=\int_{\mathbb{R}^{n}} M\left(\phi_{w}^{-1}(\mathcal{J})\right) d G(w),
$$

where $\mathcal{J} \subseteq \mathcal{Y}$ and $X(\omega) \in \phi_{w}^{-1}(\mathcal{J})(\omega)$ if and only if $\phi(X, w)(\omega) \in \mathcal{J}$, where $\omega \in \Omega$.

Theorem 1 states an invariance property of SMPPs with respect to a very general class of transformations $\Phi$. Its relevance in the present setting is due to its role in deriving a conditional estimates of SMPPs realizations. In this respect, let us consider three regions $\mathcal{I} \subseteq \mathcal{X}$ and $\mathcal{J}, \mathcal{H} \subseteq \mathcal{Y}$. Our objective is to estimate the number of points of $\mathbf{U}$ belonging to $\mathcal{H}$, knowing the restriction of the processes $\mathbf{S}$ and $\mathbf{U}$ to the regions $\mathcal{I}$ and $\mathcal{J}$ respectively. We first need to introduce the following quantity:

$$
\mathbf{U}_{(\mathcal{I})}(\mathcal{K}) \equiv \sum_{i \in \mathbb{N}} \mathbf{1}_{\left\{\Phi\left(X_{i}, w_{i}\right) \in \mathcal{K}\right\}} \mathbf{1}_{\left\{X_{i} \in \mathcal{I}\right\}}, \quad \mathcal{K} \in\{\mathcal{J}, \mathcal{H}\}
$$

The term $\mathbf{U}_{(\mathcal{I})}(\mathcal{K})$ provides the number of points of $\mathbf{S}$ fallen in $\mathcal{I}$ and sent by the transformation $\Phi$ into $\mathcal{K}$.

A straightforward computation proves the following result: 
Lemma 2. For a given $\mathcal{I} \subseteq \mathcal{X}, \mathbf{U}_{(\mathcal{I})}$ is a SMPP with mixing distribution $\Pi$ and baseline intensity measure

$$
M_{(\mathcal{I})}^{*}(\mathcal{J})=\int_{\mathbb{R}^{n}} M\left(\mathcal{I} \cap \Phi_{w}^{-1}(\mathcal{I})\right) d G(w) .
$$

In Cerqueti et al. (2009), the following theorem is proved:

Theorem 2. Consider $\mathcal{I}, \mathcal{J}, \mathcal{H}$ and define the event:

$$
E_{(l, m)} \equiv\left\{\mathbf{U}_{(\mathcal{I})}(\mathcal{H})=l, \mathbf{U}_{(\mathcal{I})}(\mathcal{J})=m\right\}, \quad l, m \in \mathbb{N} \cup\{0\} .
$$

We have

$$
\begin{gathered}
P\left(\mathbf{U}(\mathcal{H})=n \mid \mathbf{S}(\mathcal{I})=n^{\prime}, \mathbf{U}(\mathcal{J})=n^{\prime \prime}, E_{(l, m)}\right) \\
=\int_{0}^{\infty} \frac{\left[\lambda M_{(\mathcal{I})}^{*}(\mathcal{H})\right]^{n-l}}{(n-l) !} e^{-\lambda M_{(\mathcal{I})}^{*}(\mathcal{H})} u\left(\lambda ; \mathcal{I}, \mathcal{J}, n^{\prime}, n^{\prime \prime}, m\right) d \lambda,
\end{gathered}
$$

where

$$
u\left(\lambda ; \mathcal{I}, \mathcal{J}, n^{\prime}, n^{\prime \prime}, m\right)=\frac{\lambda^{n^{\prime \prime}-m+n^{\prime}} e^{-\lambda\left[M_{(\mathcal{I})}^{*}(\mathcal{J})+M(\mathcal{I})\right]} u(\lambda)}{\int_{0}^{\infty} \lambda^{n^{\prime \prime}-m+n^{\prime}} e^{-\lambda\left[M_{(\mathcal{I})}^{*}(\mathcal{J})+M(\mathcal{I})\right]} u(\lambda) d \lambda} .
$$

Theorem 2 also provides an estimate of the parameter $\lambda$, conditioned to the observation of the event $\left\{\mathbf{S}(\mathcal{I})=n^{\prime}, \mathbf{U}(\mathcal{J})=n^{\prime \prime}, \mathbf{U}_{(\mathcal{I})}(\mathcal{J})=m\right\}$. This fact allows us to explicitly derive the distribution of the transformed process $\mathbf{U}$, starting from the definition of the SMPP $\mathbf{S}$.

\section{Proof of Proposition 1}

The term $b_{r, s}^{(j)}$ can be explicitly written as:

$$
b_{r, s}^{(j)} \equiv \mathbb{E}\left[\mathbf{U}\left(\Delta_{r, s}^{(j)}\right) \mid \mathbf{S}\left(\mathcal{I}_{S}\right)=m_{S}, \mathbf{U}\left(\mathcal{I}_{U}\right)=m_{U}, \mathbf{U}_{\left(\mathcal{I}_{S}\right)}\left(\mathcal{I}_{U}\right)=m_{S, U}\right]
$$

Therefore, it is sufficient to prove that

$\mathbb{E}\left[\mathbf{U}\left(\Delta_{r, s}^{(j)}\right) \mid \mathbf{S}\left(\mathcal{I}_{S}\right)=m_{S}, \mathbf{U}\left(\mathcal{I}_{U}\right)=m_{U}, \mathbf{U}_{\left(\mathcal{I}_{S}\right)}\left(\mathcal{I}_{U}\right)=m_{S, U}\right]=\sum_{n=0}^{+\infty} \sum_{l=0}^{n} n \cdot \Gamma_{1}(n, l) \cdot \Gamma_{2}(n, l) \cdot \Gamma_{3}(n, l) \cdot \Gamma_{4}(n, l)$.

We replace in (34) the set $\mathcal{H}$ with $\Delta_{r, s}^{(j)}$, the set $\mathcal{I}$ with $\mathcal{I}_{S}$ and the set $\mathcal{J}$ with $\mathcal{I}_{U}$. By standard stochastic calculus, we can rewrite (37) as follows:

$$
\begin{gathered}
P\left(\mathbf{U}\left(\Delta_{r, s}^{(j)}\right)=n \mid \mathbf{S}\left(\mathcal{I}_{S}\right)=m_{S}, \mathbf{U}\left(\mathcal{I}_{U}\right)=m_{U}, \mathbf{U}_{\left(\mathcal{I}_{S}\right)}\left(\mathcal{I}_{U}\right)=m_{S, U}\right) \\
=\sum_{l=0}^{+\infty} P\left(\mathbf{U}\left(\Delta_{r, s}^{(j)}\right)=n \mid \mathbf{S}\left(\mathcal{I}_{S}\right)=m_{S}, \mathbf{U}\left(\mathcal{I}_{U}\right)=m_{U}, \mathbf{U}_{\left(\mathcal{I}_{S}\right)}\left(\mathcal{I}_{U}\right)=m_{S, U}, \mathbf{U}_{\left(\mathcal{I}_{S}\right)}\left(\Delta_{r, s}^{(j)}\right)=l\right) \times \\
\times P\left(\mathbf{U}_{\left(\mathcal{I}_{S}\right)}\left(\Delta_{r, s}^{(j)}\right)=l \mid \mathbf{S}\left(\mathcal{I}_{S}\right)=m_{S}, \mathbf{U}\left(\mathcal{I}_{U}\right)=m_{U}, \mathbf{U}_{\left(\mathcal{I}_{S}\right)}\left(\mathcal{I}_{U}\right)=m_{S, U}\right),
\end{gathered}
$$

Therefore, by Theorem 2, we can rewrite (38) as follows:

$$
\begin{aligned}
P\left(\mathbf{U}\left(\Delta_{r, s}^{(j)}\right)\right. & \left.=n \mid \mathbf{S}\left(\mathcal{I}_{S}\right)=m_{S}, \mathbf{U}\left(\mathcal{I}_{U}\right)=m_{U}, \mathbf{U}_{\left(\mathcal{I}_{S}\right)}\left(\mathcal{I}_{U}\right)=m_{S, U}\right)= \\
& =\sum_{l=0}^{n} \Gamma_{1}(n, l) \cdot \Gamma_{2}(n, l) \cdot \Gamma_{3}(n, l) \cdot \Gamma_{4}(n, l) .
\end{aligned}
$$


The thesis is a consequence of (39), by recalling that

$$
\begin{gathered}
\mathbb{E}\left[\mathbf{U}\left(\Delta_{r, s}^{(j)}\right) \mid \mathbf{S}\left(\mathcal{I}_{S}\right)=m_{S}, \mathbf{U}\left(\mathcal{I}_{U}\right)=m_{U}, \mathbf{U}_{\left(\mathcal{I}_{S}\right)}\left(\mathcal{I}_{U}\right)=m_{S, U}\right]= \\
=\sum_{n=0}^{+\infty} n \cdot P\left(\mathbf{U}\left(\Delta_{r, s}^{(j)}\right)=n \mid \mathbf{S}\left(\mathcal{I}_{S}\right)=m_{S}, \mathbf{U}\left(\mathcal{I}_{U}\right)=m_{U}, \mathbf{U}_{\left(\mathcal{I}_{S}\right)}\left(\mathcal{I}_{U}\right)=m_{S, U}\right) .
\end{gathered}
$$

